A Bayesian Approach to Detect Pedestrian Destination-Sequences from WiFi Signatures

\author{
Antonin Danalet \\ Bilal Farooq \\ Michel Bierlaire
}

TRISTAN VIII, San Pedro de Atacama June $10^{\text {th }}, 2013$ 


\section{Presentation outline}

- Motivation

- Data requirement

- Methodology

- A case study on EPFL campus

- Conclusion

- Future work 


\section{MOTIVATION}




\section{Walking is the key for efficient multimodal transport systems}

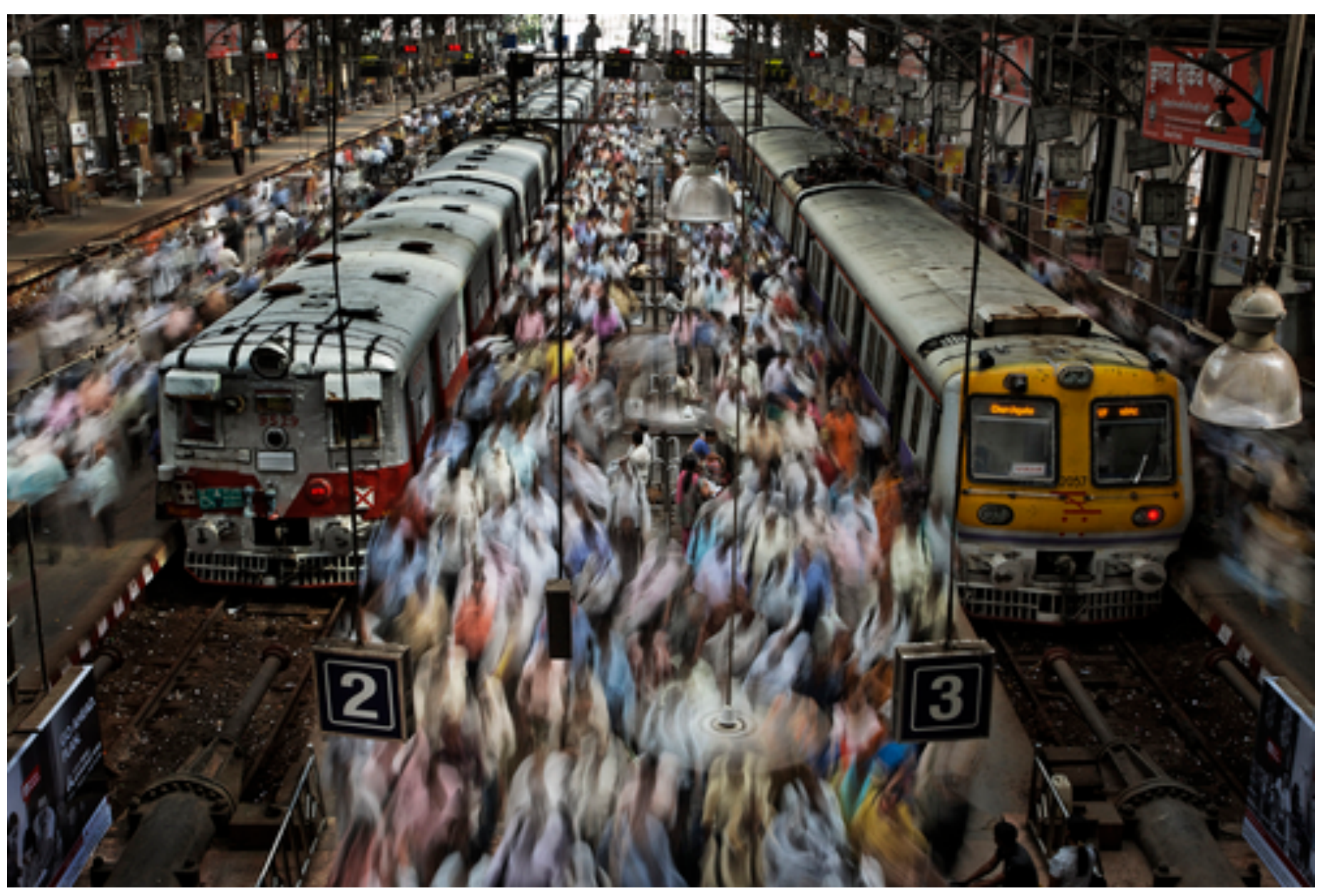

Crowd in a railway station in Mumbai, India Photo: National Geographic 


\section{Lake Geneva region}

$>25^{\prime} 000$ travellers/day between Geneva and Lausanne*

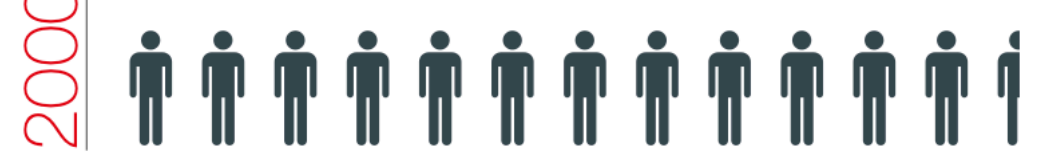

$>50^{\prime} 000$ travellers/day between Geneva and Lausanne*

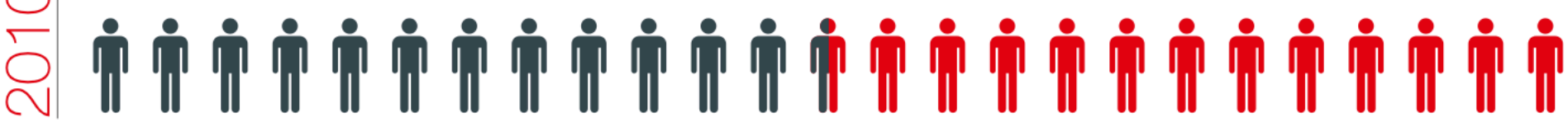

$>100^{\prime} 000$ travellers/day between Geneva and Lausanne*

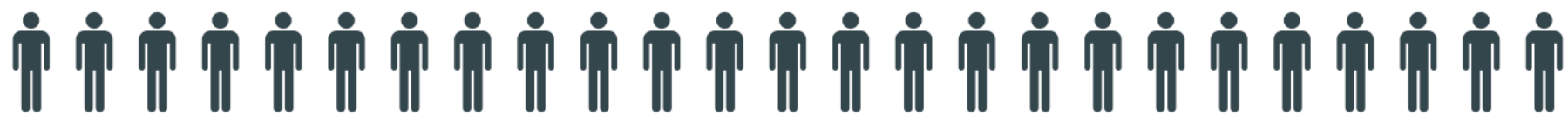

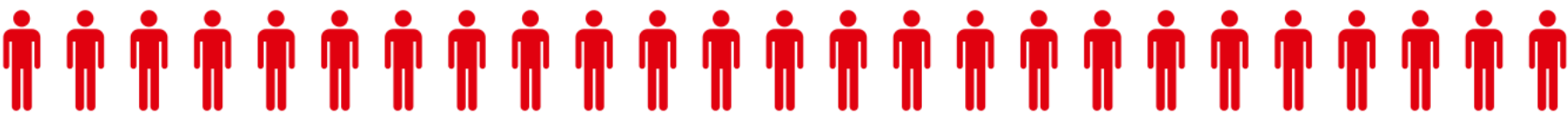

$\tilde{\|}=2000$ travelers/day $\quad$ * Forecast by Swiss Railways for the maximum scenario
By 2030, $100{ }^{\prime} 000$ passengers per day between Geneva and Lausanne 


\section{Understand pedestrian activities}

What we are doing: Campus

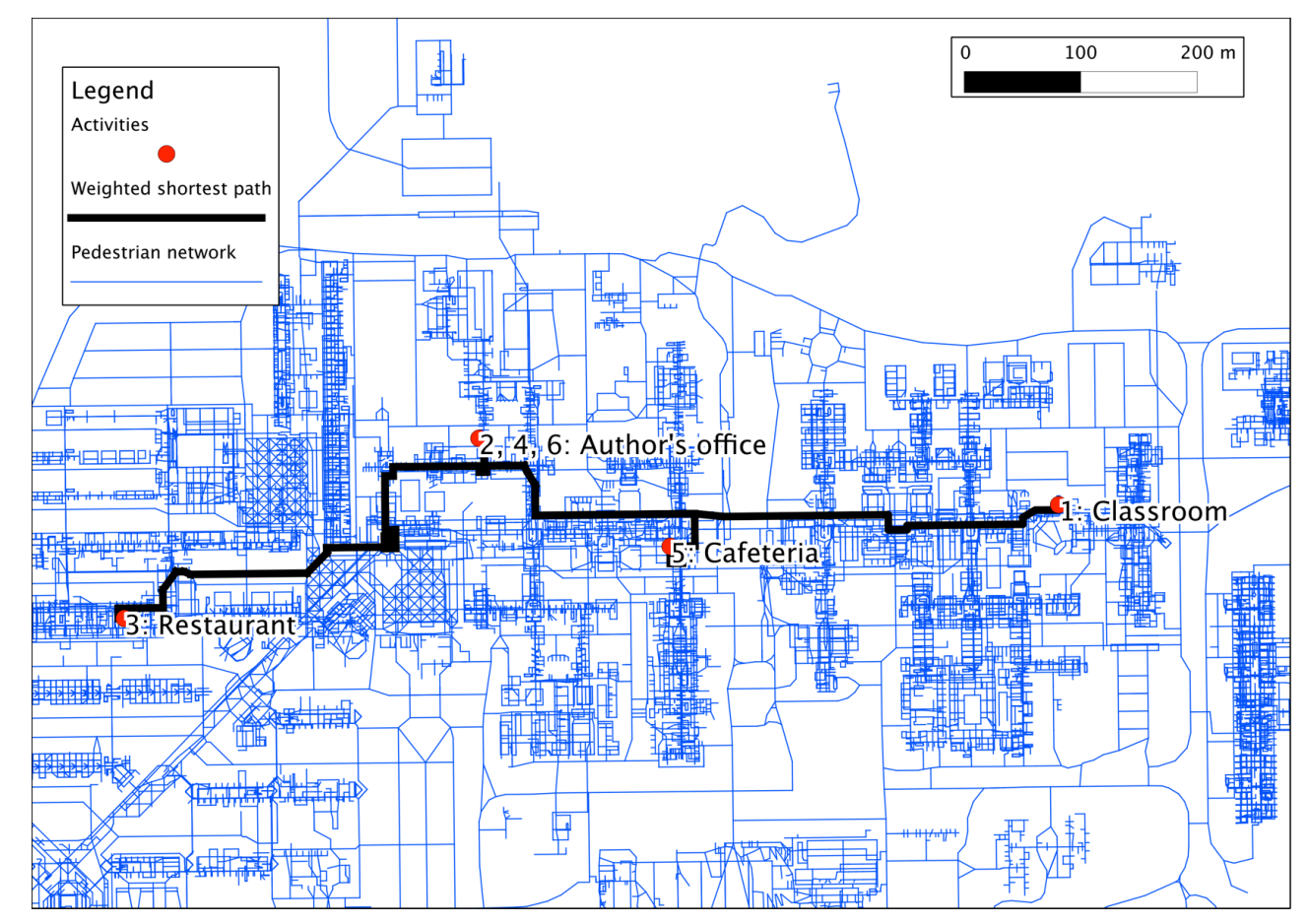

\section{What we want to do: Station}

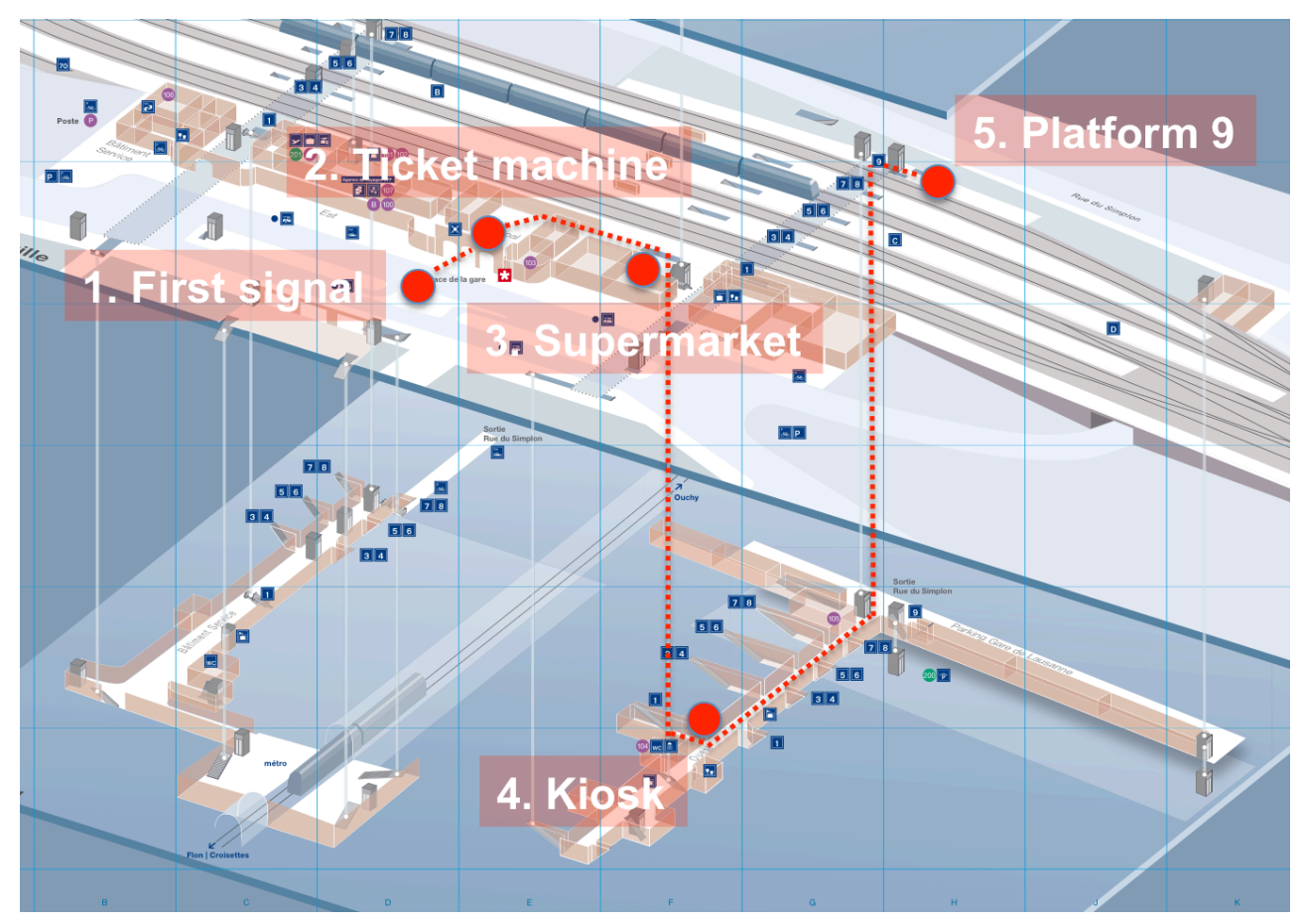




\section{Challenges}

- Detect pedestrian destinations

- Model pedestrian activity scheduling behavior

- Forecast the impact of changes in the infrastructure

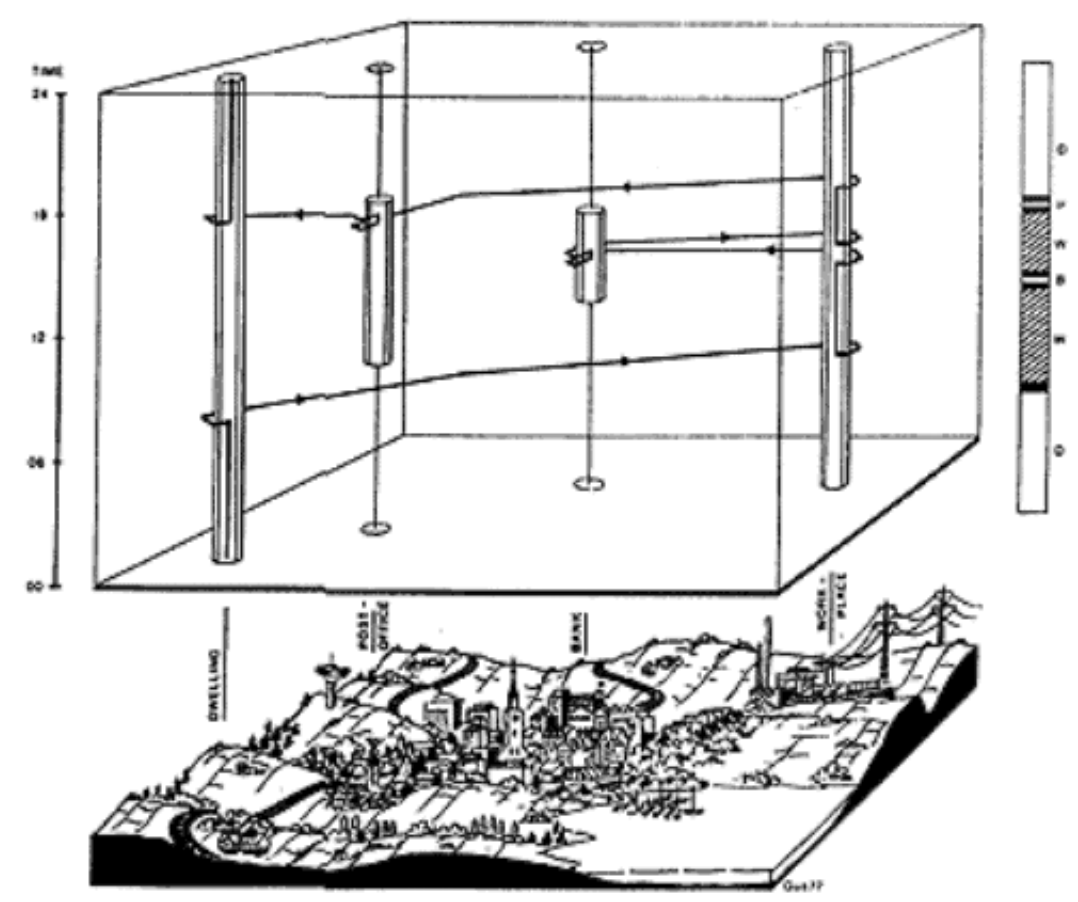

Carlstein, T. (1978) 


\section{DATA REQUIREMENT}




\section{Data requirement}

- Required

- Localization data with full coverage of the facility

- Semantically-enriched routing graph for pedestrians

- Not really required but often available information

- Prior potential attractivity 


\section{Data requirement: Localization}

- Data from communication network infrastructure

- GSM traces (Calabrese et al. 2011, Bekhor et al. 2011)

- WiFi traces

- Data processing is needed (Rieser-Schüsseler 2012)

- Detection of stop points

- Activity purpose detection through land-use information and spatial matching 


\section{Data requirement: Pedestrian network}

- We need maps

- With points of interests (space)

- With shortest path (time)

- More and more available in airports, malls, museums, campuses, hospitals

- Nokia: 214 shopping malls in 2011, 4605 indoor maps in July 2012, 5100 in December 2012

- Microsoft: 2700 indoor maps

- Google: > 10'000 indoor maps

- Start-ups: Wifarer, Meridian, Point Insider, ByteLight 


\section{Data requirement: Potential attractivity}

- Potential attractivity $\mathbf{C}(\mathbf{x}, \mathbf{t})$ depends on

- destination $x$

- Classroom, platform, scene, ...

- time $\mathbf{t}$

- class schedules, train schedules, opening hours, ...

- Examples:

- 1500 passengers on platform 4 arriving at $16 \mathrm{~h} 04$

- 32 students in a classroom from $8 \mathrm{~h} 15$ to $10 \mathrm{~h}$

- 400 seats in a restaurant open from $11 \mathrm{~h}$ to $14 \mathrm{~h} 30$ 


\section{METHODOLOGY}




\section{Methodology}

- Goal: extract the possible activity-episodes performed by pedestrians from digital traces from communication networks

- Localization measurement

- Potential attractivity

- Semantically-enriched routing graph

\section{Input}

\section{Output}

- set of candidate activityepisodes sequences associated with the likelihood to be the true one 


\section{Definitions / Notations}

- Measurement: $\hat{s}=(\hat{x}, \hat{t})$

- Activity-episode: $a=\left(x, t^{-}, t^{+}\right)$

- Episode location, start time and end time

- Activity-episode sequence: $\left(a_{1}, \ldots, a_{m}\right)=a_{1: m}$

- Activity: $A(a)$

- Activity pattern: $\left(A_{1}, \ldots, A_{m}\right)=A_{1: m}$ 


\section{Methodology}

- Probabilistic measurement model:

A Bayesian approach

- Measurement equation

- Prior

- Generation of activity-episode sequences

- Episode location

- Episode start and end times 


\section{Probabilistic measurement model}

Measurement likelihood
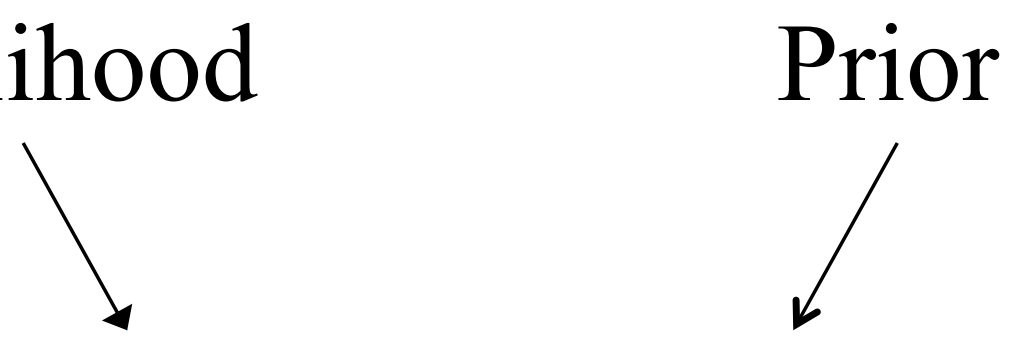

$P\left(a_{1: m} \mid \hat{s}_{1: n}\right) \propto P\left(\hat{s}_{1: n} \mid a_{1: m}\right) \cdot P\left(a_{1: m}\right)$

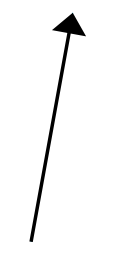

Activity model 


\section{Measurement error}

$$
\begin{aligned}
P\left(\hat{s}_{1: n} \mid a_{1: m}\right) & =\prod_{j=1}^{m} P\left(\hat{s}_{i_{j-1}+1: i_{j}} \mid a_{j}\right) \longleftarrow \text { Independence between activities } \\
& =\prod_{j=1}^{m} \prod_{i=1}^{n} P\left(\hat{s}_{i_{j}} \mid a_{j}\right) \longleftarrow \text { Independence between signals } \\
& =\prod_{j=1}^{m} \prod_{i=1}^{n} P\left(\hat{x}_{i_{j}} \mid x_{j}\right) \longleftarrow \text { No time measurement error }
\end{aligned}
$$




\section{Prior}

$$
\begin{aligned}
P\left(a_{1: m}\right) & =\prod_{j=1}^{m} P\left(a_{j}\right) \\
& =\prod_{j=1}^{m} P\left(x_{j}, t_{j}^{-}, t_{j}^{+}\right) \\
& =\prod_{j=1}^{m} \frac{C_{x_{j}}\left(t_{j}^{-}, t_{j}^{+}\right)}{\sum_{x \in X} C_{x}\left(t_{j}^{-}, t_{j}^{+}\right)} \\
& =\prod_{j=1}^{m} \int_{t_{j}=t_{j}^{-}}^{t_{j}^{+}} \frac{C_{x_{j}}\left(t_{j}\right)}{\sum_{x} C_{x}\left(t_{j}\right)} d t
\end{aligned}
$$




\section{Generation of activity-episode sequences}

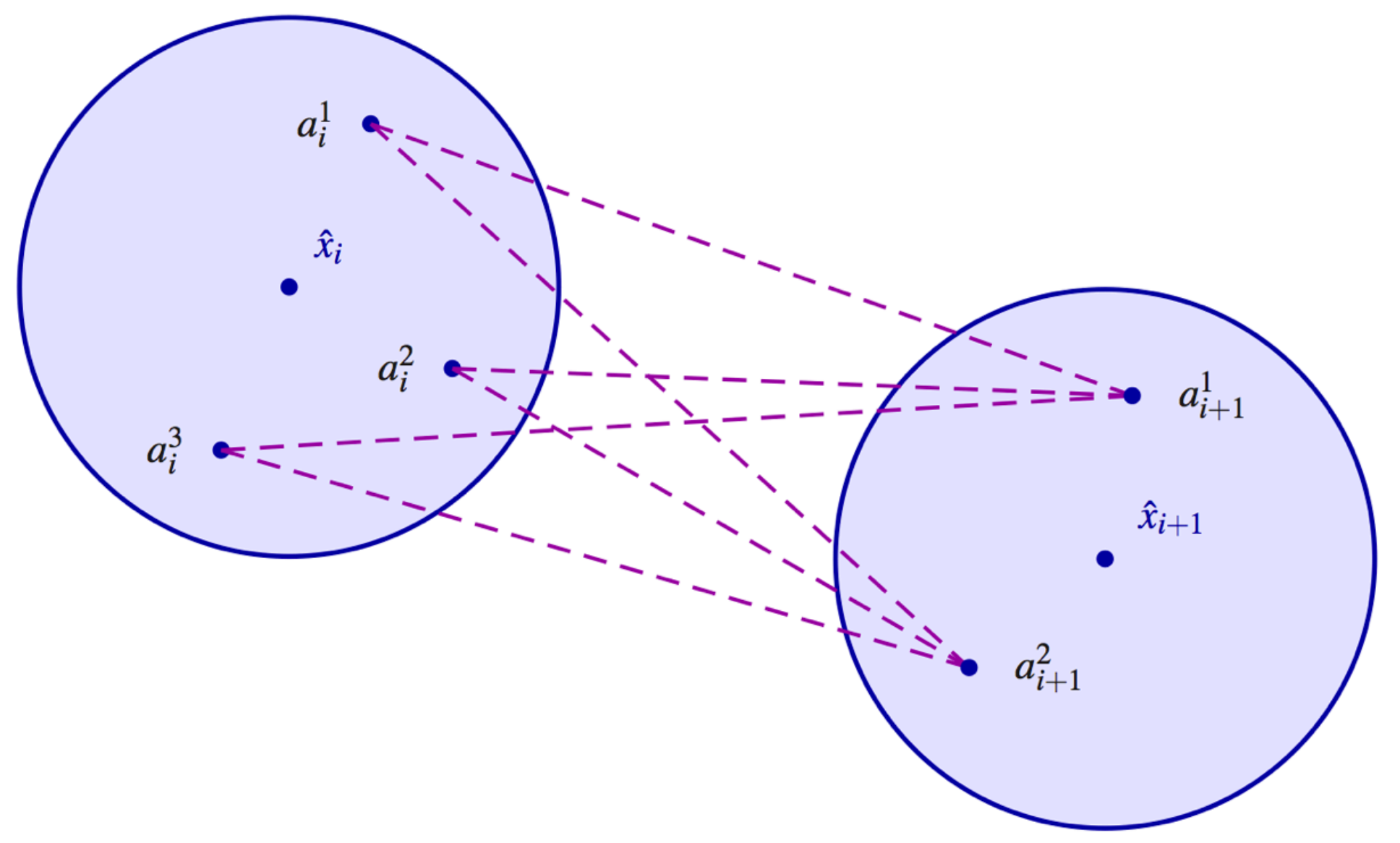




\section{Generation of activity-episode sequences}

\section{Position}

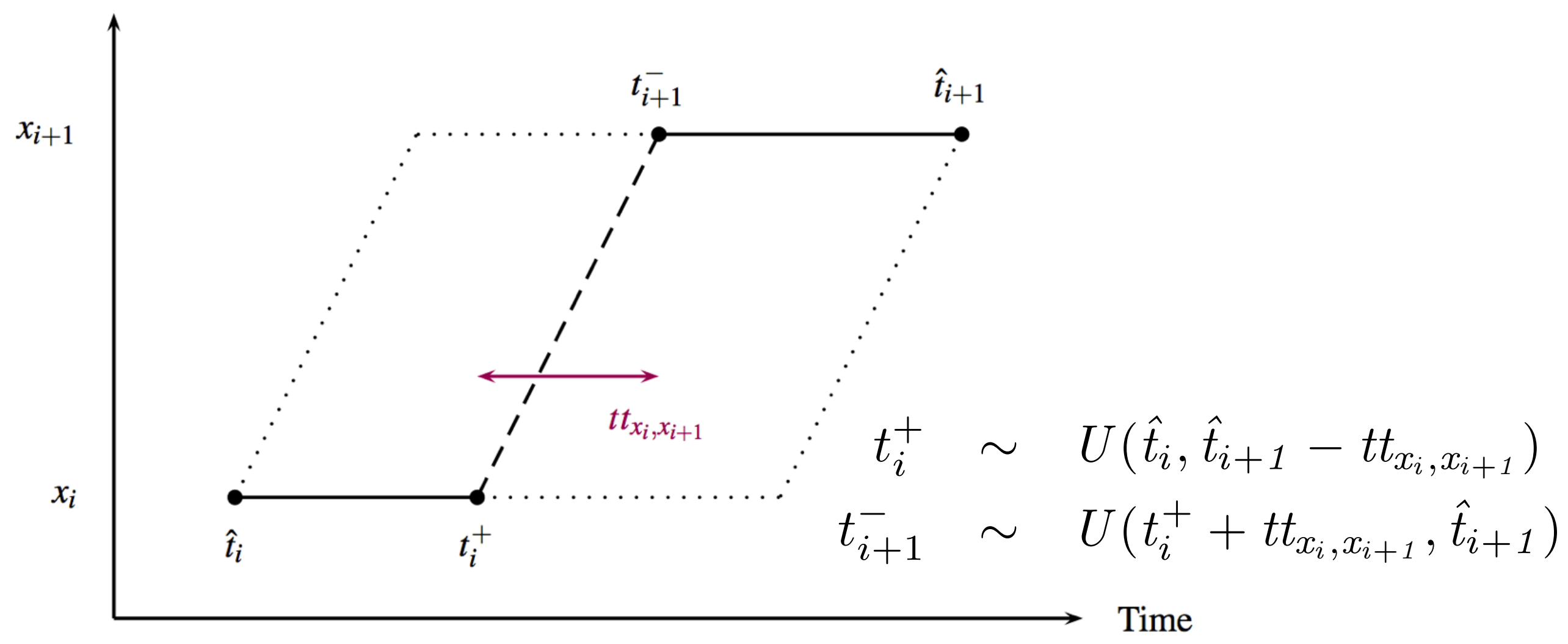




\section{Generation of activity-episode}

\section{sequences}

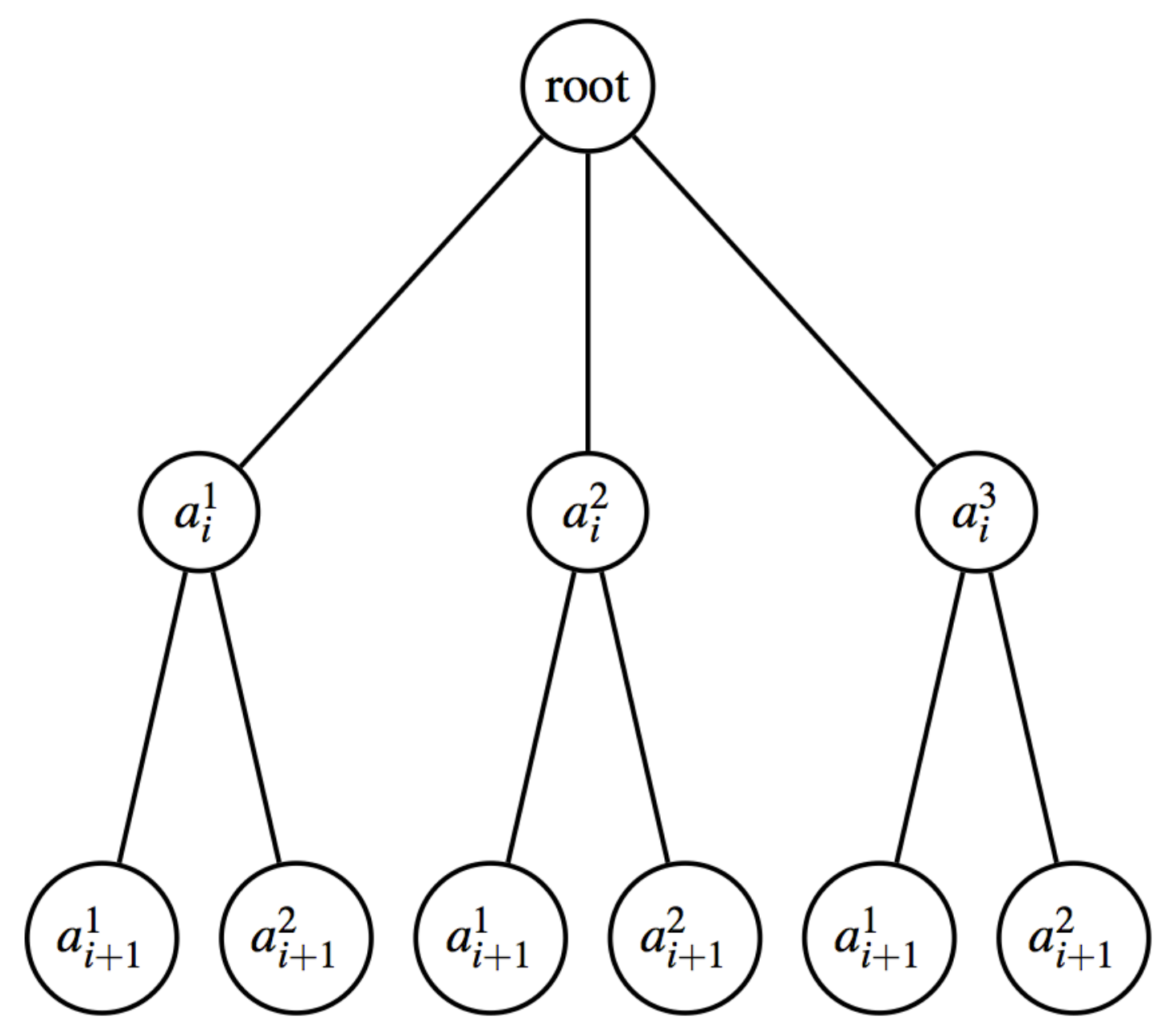




\section{Intermediary signals}

- Eliminate intermediary signal if

$$
E\left(t^{+}\right)-E\left(t^{-}\right)<T_{\min }
$$

since we generate an activity episode at each signal. 


\section{Sequence elimination}

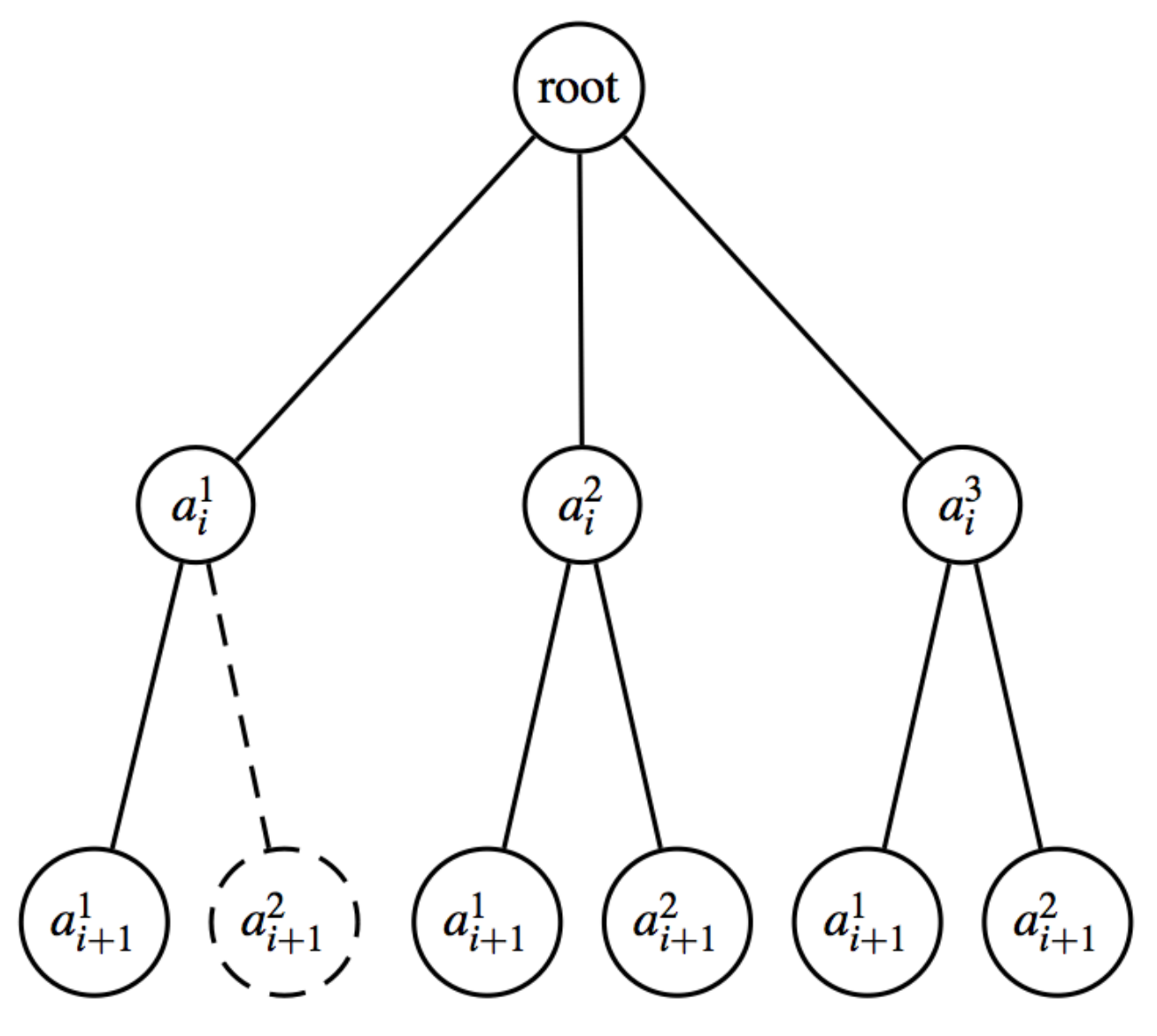

$\begin{array}{llllll}0.1 & 0.05 & 0.3 & 0.2 & 0.15 & 0.2\end{array}$ 


\section{A CASE STUDY ON EPFL CAMPUS}




\section{EPFL data: Localization}

- 8 participants for 2 months with known ID

- Non-participants: 46 days, but only 10 with courses

- 200 students in 6 different classes

- 317 employees

- 700 students from

University of Lausanne

- For $151 \mathrm{CE}$ students, 152'598 observations

- Precision: 191 meters

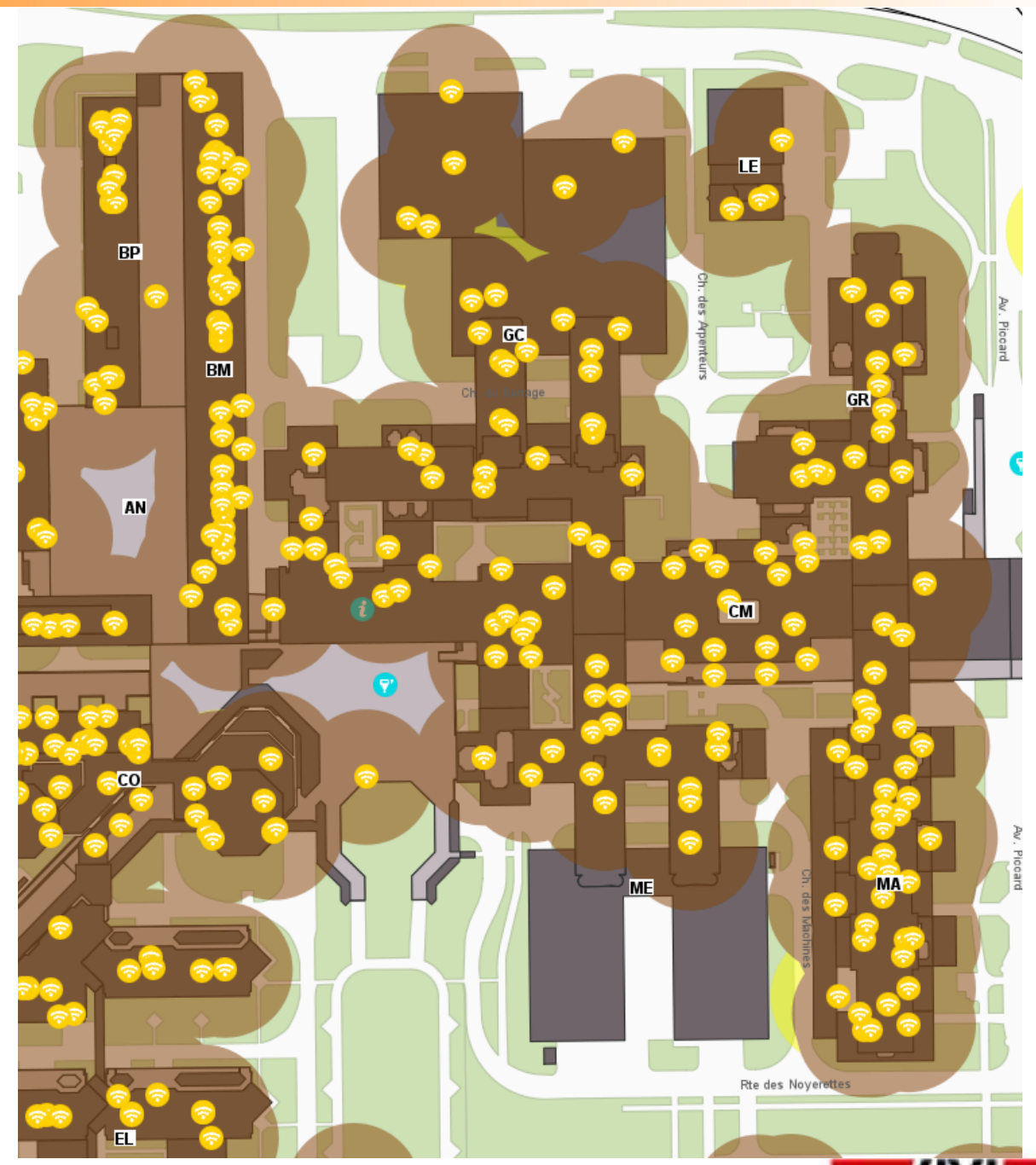




\section{EPFL data: Pedestrian network}

- Source: map.epfl.ch

- 56’655 edges

- 4 different levels of path

- Major (« highway »)

- Inter-building

- Intra-building

- Access to offices

- Shortest path

- All offices, restaurants, classrooms and other points of interest are coded: $X$

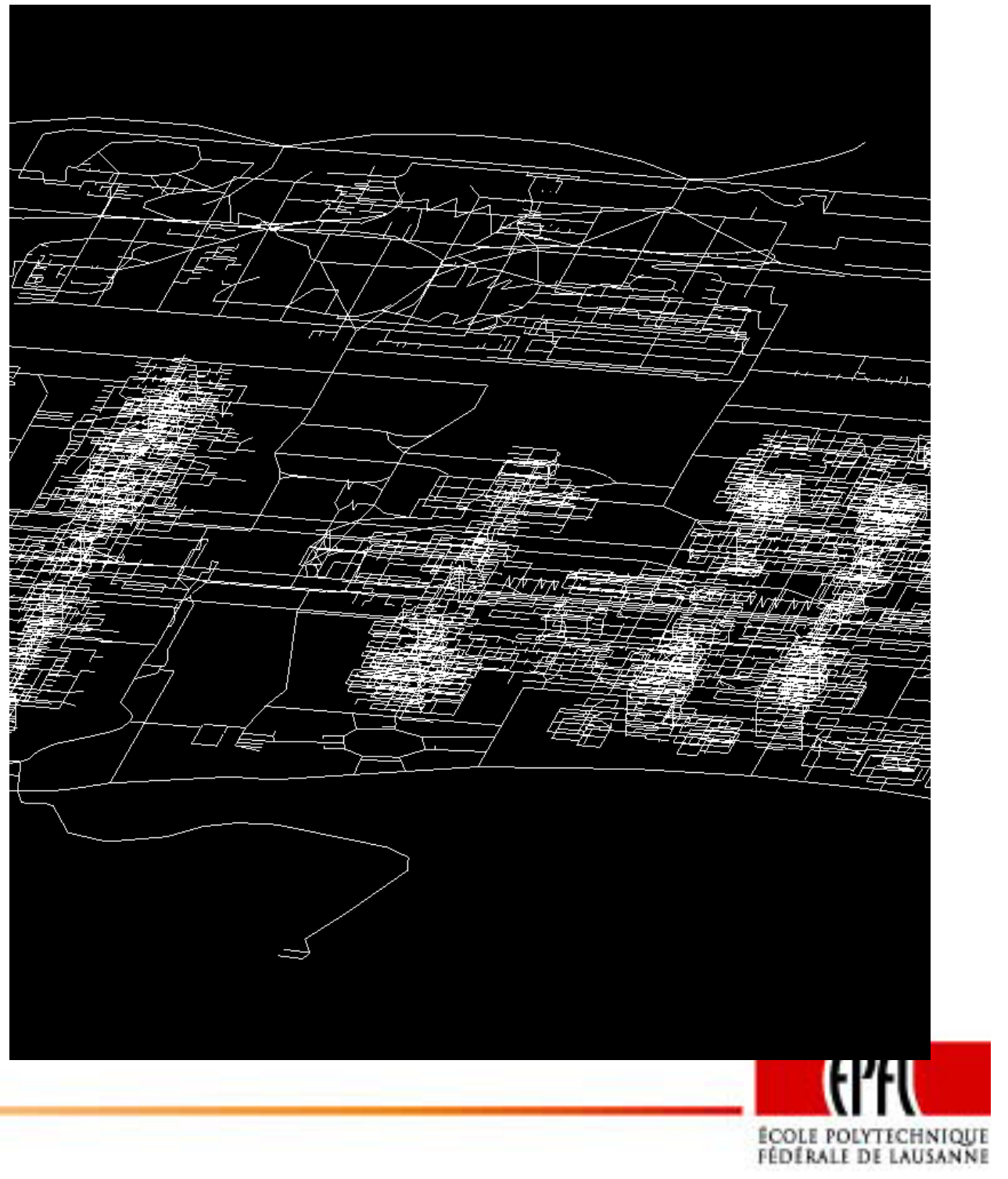

TRANSP-OR 


\section{EPFL data: Potential attractivity $\mathbf{C}(\mathbf{x}, \mathbf{t})$}

- Class schedules with

- Number of students

- Name of the classroom

- Number of employees per office

- Name of the office

- Sum of percent of work (e.g, 3 full times $=300 \%$ )

- Number of seats in restaurants

- Localization

- Opening hours

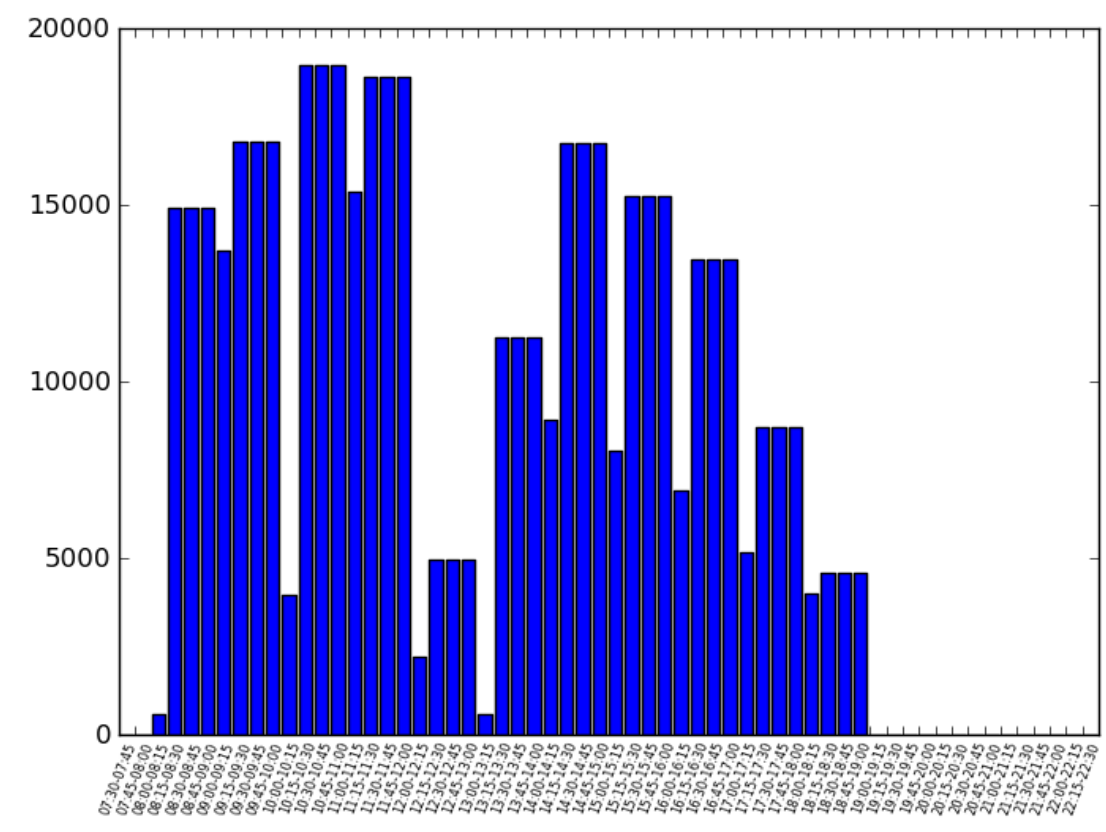

- Number of seats in library 


\section{Results}

Model
\begin{tabular}{|l|l|c|l|l|c|l|c|}
\hline Arrival time & Departure time & Floor & Location & Time spent & Floor & Location & $\Delta x$ \\
\hline $8: 33-8: 33$ & $10: 38-10: 38$ & 1 & Classroom & 8.32am-10.30am & 1 & Classroom & 0 \\
\hline 10:40-10:40 & $11: 51-11: 51$ & 3 & Office & Until 11.47am & 3 & Author's office & 7 \\
\hline $11: 54-11: 54$ & $12: 47-12: 53$ & 1 & Restaurant & From 11.55 am & 1 & Restaurant & 0 \\
\hline 12:51-12:58 & $13: 03-13: 44$ & 3 & Office & Around 1pm & 3 & Author's office & 7 \\
\hline $13: 06-13: 47$ & $13: 53-14: 02$ & 2 & Cafeteria & Around 2pm & 2 & Cafeteria & 0 \\
\hline 13:55-14:04 & $19: 45-19: 45$ & 3 & Office & Until around 7.45pm & 3 & Author's office & 7 \\
\hline 19:47-19:47 & $19: 52-19: 52$ & 3 & Workshop & - & 3 & Metro stop & 366 \\
\hline
\end{tabular}




\section{SENSITIVITY ANALYSIS}




\section{Sensitivity analysis: prior}

- With flat prior

- \# destinations / Start and end time: OK

- Distance / category of destination: Not OK

- Attractivity of visited destinations should be $3 \mathrm{x}$ bigger than of non-visited destinations

- Global capacity creates bias 


\section{CONCLUSION}




\section{Conclusion}

- Prior needed to overcome low precision

- Localization data brings dynamics in the model

- Pedestrian map gives:

- Spatial information

- Temporal information

- Our methodology is merging these different types of data

- Robust for low density data 


\section{FUTURE WORK}




\section{Future work}

- Binary choice model for attendance of scheduled activity

- Actual start and end times of scheduled activityepisodes

- Analysis of the access to the facility

- First and last destination of the sequence

- Arrival times, departure times

- Based on class attendance and on available time budget: activity scheduling 


\section{THANK YOU}




\section{References}

- Bekhor, S., Cohen, Y. and Solomon, C. (2011), Evaluating long-distance travel patterns in Israel by tracking cellular phone positions. J. Adv. Transp.. doi: 10.1002/atr.170

- Calabrese, F.; Di Lorenzo, G.; Liang Liu; Ratti, C., "Estimating OriginDestination Flows Using Mobile Phone Location Data," Pervasive Computing, IEEE , vol.10, no.4, pp.36,44, April 2011 doi: 10.1109/MPRV.2011.41

- Rieser-Schüssler, N. (2012). Capitalising Modern Data Sources for Observing and Modelling Transport Behaviour, Transportation Letters: the International Journal of Transportation Research (2): 115-128. doi: 10.3328/TL.2012.04.02.115-128 